\title{
Additions and corrections to the Catalogue of Palaearctic Coleoptera, vol. 6/1, 2020. Revised and Updated Second Edition. Chrysomeloidea I (Vesperidae, Disteniidae, Cerambycidae)
}

\section{Аополнения и исправления к каталогу палеарктических Coleoptera, том 6/1, 2020. Пересмотренное и обновлённое второе издание. Chrysomeloidea I (Vesperidae, Disteniidae, Cerambycidae)}

\author{
M.L. Danilevsky

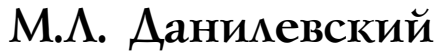

\begin{abstract}
A. N. Severtzov Institute of Ecology and Evolution, Russian Academy of Sciences, Leninsky prospect 33, Moscow 119071, Russia. E-mail: danilevskyml@rambler.ru,danilevsky@cerambycidae.net

Институт Проблем Экологии и Эволюции им. А.Н. Северцова РАН, Ленинский пр. 33, Москва 119071, Россия.
\end{abstract}

\begin{abstract}
KEY WORDS: Cerambycidae, taxonomy, new combinations, new synonyms, new names, Palaearctic.
КЛЮЧЕВЫЕ СЛОВА: Cerambycidae, таксономия, новые сочетания, новые названия, Палеарктика.
\end{abstract}

ABSTRACT. About 60 corrections are published for Volume 6/1 of the Catalogue of Palaearctic Coleoptera [Danilevsky, 2020 (ed.)]. The geographical limits of Russian East and West Siberia must be modified. All taxa from Tuva Republic of Russia were marked in the text with symbol "ES" (East Siberia), while south Tuva is in the West Siberia in the map of Russian regions (p. XIX), so the map must be corrected. Several misprints, wrong spellings of the taxa names, wrong combinations, missing or wrong geographical symbols, wrong dates of original descriptions are fixed. Original genus names were missing in several species; several references were published with wrong dates; several taxonomy actions published as new were published before. A valid name Anaglyptus (s.str.) arakawae arakawae Kano, 1933a was published as a synonyme. The synonyms: Dorcadion semenowi var. is sykkulense Pic, 1906c = Dorcadion bisbicostatum Pic, 1908 e published in the list of taxa, were not marked as new, and are discussed below. Purpuricenus sasanus Kadlec, 2006a was originally published as a species. Then its status was downgraded to subspecies rank by Sama and Löbl [2010], Rapuzzi and Sama [2013]. The species rank was restored without comments. It was upgraded to species rank by Danilevsky et al. [2020]. An available name Oberea alexandrovi ssp. infrequens Cherepanov, 1996 was missing. An unavailable name Saperda praecestus Dufour, 1843 was published as available.

РЕЗЮМЕ. Для нового каталога жуков-усачей Палеарктики [Danilevsky, 2020 (ed.)] приводится около 60 исправлений. Уточнены границы Западной и
Восточной Сибири. Все таксоны из Тувинской Республики России помечены в тексте символом "ES" (Восточная Сибирь), в то время как южная Тува отнесена к Западной Сибири на карте регионов России (стр. XIX); таким образом, карта должна быть исправлена. Исправлены несколько опечаток, ошибочные написания названий таксонов, ошибочные сочетания, ошибочные даты оригинальных описаний, добавлены пропущенные географические символы и указаны лишние. Для некоторых названий видовой группы приведены ошибочные оригинальные названия родов. Несколько литературных ссылок напечатаны с ошибочными датами. Несколько таксономических преобразований ошибочно приведены как новые, хотя они публиковались раньше. Валидное название Anaglyptus (s.str.) arakawae arakawae Kano, 1933а было ошибочно опубликовано, как синоним. Новые синонимы Dorcadion semenowi var. issykkulense Pic, 1906c = Dorcadion bisbicostatum Pic, 1908е, опубликованные в списке таксонов, не помечены как новые; они аргументированы в настоящей статье. Purpuricenus sasanus Kadlec, 2006а был опубликован первоначально как вид. Затем ранг названия был понижен до подвидового [Sama, Löbl, 2010; Rapuzzi, Sama, 2014b]. Видовой статус возвращён в каталоге без комментариев, хотя был восстановлен ранее [Danilevsky et al., 2020]. В каталоге пропущено одно пригодное название Oberea alexandrovi ssp. infrequens Cherepanov, 1996. Одно непригодное название - Saperda praecestus Dufour, 1843 - опубликовано как пригодное. 


\section{Introduction}

The new Catalogue of Palaearctic Chrysomeloidea I (Coleoptera) edited by Danilevsky [2010] was prepared with the participation of 13 authors: K. Adlbauer, M. L. Danilevsky, A. Drumont, H. K. Jang, Z. Komiya, M. A. Lazarev, Seunghyun Lee, J. Lim, M.-Y. Lin, T. Niisato, S.-H. Oh, N. Ohbayashi and J. Yamasako. The system of the authorship was as much complicated as in the previous Catalogue [Löbl, Smetana (ed.), 2010]. It was partly arranged in taxonomy way and partly in geographical. So, such groups as Prioninae (by A. Drumont and Z. Komiya) or Dorcadionini (by M. L.Danilevsky) had own authors, while other authors were responsible for a certain geographical areas. The taxa of Korea, for example, were arranged by H.K. Jang, Seunghyun Lee, J. Lim and S.-H. Oh, while T. Niisato, N. Ohbayashi and J. Yamasako were responsible for the taxa of Japan. So, the authorship of all Cerambycidae genera, which are distributed in Korea and in Japan was in fact mutual. Each author had his own point of view on the system of each subfamily and each genus. So, the editor had to create a generally accepted system on the base of several different positions.

The main purpose of the present publication is to fix as many misprints and mistakes, as possible.

The references to the present article include only the publications absent in the references to the Catalogue. The references inside the text of the present article to the publications included in the references to the Catalogue have same letters after the number of the year as in the Catalogue.

All changes (additions, corrections and etc.) are underlined. Moreover, all of them are regularly published and updated in http://cerambycidae.net/.

\section{PAGE XVI}

PRINTED:

Each country is marked with two-letters symbol, as well as major parts of Russia and North India.

MUST BE:

Each country is marked with two-letters symbol, as well as major parts of Russia and North India. Tuva Republic of Russia was divided in two parts in all editions of the Catalogue, a part northwards Enisey River (with Kaa-Hem) was in the East Siberia [ES], and a south part southwards Enisey River (with Kaa-Hem) was in the West Siberia [WS]. Now the whole Tuva territory is regarded as a part of East Siberia.

\section{PAGE XXI}

PRINTED:

HEN Henan (Honana)

MUST BE:

HEN Henan (Honan)

\section{PAGE 1}

PRINTED AS NEW SYNONYMS:

Diboma bhutana Breuning, 1975a $($ March $)=$ Diboma bhutanensis Breuning, 1975d (October), syn. nov. (now in Zotalemimon Pic, 1925a)

The synonyms were published before by Danilevsky [2014b: 221].

\section{PAGE 2}

A proposal of a new synonym was missing.

MUST BE PUBLISHED:

Dorcadion semenowi var. issykkulense Pic, $1906 \mathrm{c}=$ Dorcadion bisbicostatum Pic, 1908e, syn. nov. Before [Danilevsky, 2002; 2010ñ; Toropov, Milko, 2013] Dorcadion semenovi bisbicostatum Pic, 1908d was accepted as a valid name of a subspecies from Narynkol valley in Kirgizia. Two syntypes are preserved in Pic's collection in Paris Muséum national d'Histoire naturelle. No other specimens of $D$. semenovi were ever collected in the region. Most probably both syntypes were supplied with wrong labels and were collected near Issyk-Kul Lake, as well as so labeled types of "Dorcadion robustissimum Pic, 1908", which were quite same as $D$. (s.str.) crassipes validipes Jak. from near Issyk-Kul. D. bisbicostatum Pic, $1908 \mathrm{~d}$ was accepted as a synonym of $D$. semenovi by Plavilstshikov [1958a] and Breuning [1962a].

\section{PAGE 2}

PRINTED AS NEW SYNONYMS:

Dorcadion theophilei Pic, $1898 \mathrm{~h}=$ D. kadleci Bernhauer \&

Peks, 2016, syn. nov. based on the original description.

The synonyms were published before by Danilevsky [2017d].

\section{PAGE 2}

PRINTED:

Aromia moschata rosara P.H. Lucas, 1847

MUST BE:

Aromia moschata rosarum P.H. Lucas, 1847

According to the original description.

\section{PAGE 3}

PRINTED AS A TAXONOMICAL NEWS:

Dorcadion theophilei costiferum Pic, 1898 is a valid name of a subspecies from Trabzon province.

That opinion was published before by Danilevsky [2017d].

\section{PAGE 3}

PRINTED AS A TAXONOMICAL NEWS:

Phytoecia (Musaria) puncticollis persica Ganglbauer, 1884 The validity of the name was accepted before by Fuchs, Breunng [1971] and then by Sama [1982], Rejzek et al. [2003], Özdikmen [2007], Özdikmen, Turgut [2007].

\section{PAGE 4}

PRINTED AS A TAXONOMICAL NEWS:

Dorcadion lohsei taskentense Bernhauer \& Peks, 2016, D. wagneri karayaziense Bernhauer \& Peks, 2016, D. sodale soganliense Bernhauer \& Peks, 2016, D. theophilei kostandagense Bernhauer \& Peks, 2016, D. jacovleviellum hinisense Bernhauer \& Peks, 2016, and D. jacovleviellum vartoense Bernhauer \& Peks, 2016 were accepted.

All subspecies were accepted before [Danilevsky, 2017d].

\section{PAGE 7}

PRINTED:

Lamia lusitanicus Olivier, 1790b

MUST BE:

Callidium lusitanicum Olivier, 1790b

\section{PAGE 15}

PRINTED:

body length in male: $311.5-20.0 \mathrm{~mm}$

MUST BE:

body length in males: 11.5-20.0 mm 


\section{PAGE 119}

PRINTED:

bicolor K. Ohbayashi, 1941a: 1 A: JA

MUST BE:

bicolor K. Ohbayashi, 1941a: 1 A: JA SC

\section{PAGE 122}

PRINTED:

atra fabricius, 1775: 197 (Leptura) [NO]

MUST BE:

atra Fabricius, 1775: 197 (Leptura) [NO]

\section{PAGE 155}

PRINTED:

balcanica Hampe, 1871: 336 E: AL BU GR RO SB TR A: TR bureschi Kantardjiewa-Minkowa, 1957: 542

MUST BE:

balcanica Hampe, 1871: 336 E: AL BU GR RO SB ?TR A: TR bureschi Kantardjiewa-Minkowa, 1957: 542 (Evodinus)

15. PAGE 166

PRINTED:

sichotensis Danilevsky, 1988c: 372 A: FE

MUST BE:

sichotensis Danilevsky, 1988c: 372 A: FE KO

16. PAGE 180

PRINTED:

koltzei Heyden, 1887c: 304 (Brachyta) A: ES FE GUA HEI JA LIA NC NMO SC

MUST BE:

koltzei Heyden, 1887a: 304 (Brachyta) A: ES FE GUA HEI JA LIA NC NMO SC

\section{PAGE 187}

PRINTED:

sudeticus C.F.W. Richter, 1820: pl. 10 (Prionus)

MUST BE:

sudeticus C.F.W. Richter, 1821: pl. 10 (Prionus)

18. PAGE 191

PRINTED:

colobotheoides Bates, 1884a: 235 (Aglaophis) A: FE JA NC

NE QIN SC \#400

angustefasciatus Heyden, 1884: 297 (Aglaophis)

arakawai Kano, 1933a: 276

MUST BE:

colobotheoides Bates, 1884a: 235 (Aglaophis) A: FE JA NC

NE QIN SC \#400

angustefasciatus Heyden, 1884: 297 (Aglaophis)

arakawai Kano, 1933a: 276

Anaglyptus (s.str.) arakawae arakawae Kano, 1933a: 276 (Aglaophis) A: JA

is a valid name (see p. 192).

19. PAGE 198

PRINTED:

moschata ambrosiaca Steven, 1809: 40 (Cerambyx) E: IT PT

SP ST TR N: AG MO TU A: AB AR

GG IN IQ JO LE SY TR \#133 \#400

melancholica Reitter, 1895d: 210

notaticollis Pic, 1928c: 9

rosara Costa, 1855: 26 [HN]

MUST BE: moschata ambrosiaca Steven, 1809: 40 (Cerambyx) E: IT PT SP ST TR N: AG MO TU A: AB AR

GG IN IQ JO LE SY TR \#133 \#400

melancholica Reitter, 1895d: 210

notaticollis Pic, 1928c: 9

rosarum Costa, 1855: $26[\mathrm{HN}]$

as in the original description.

20. PAGE 209

PRINTED:

clavipes Fabricius, 1775: 188 (Callidium) E: AL AU BE BH BU BY CR CT CZ DE FR GE GR HU IT LA LT LU MA MC MD ME NL NT PL RO SB SK SL SP ST SZ TR UK A: AB AR GG FE

IN KZ IQ TR WS

MUST BE:

clavipes Fabricius, 1775: 188 (Callidium) E: AL AU BE BH BU BY CR CT CZ DE FR GE GR HU IT ŁALT LU MA MC MD ME NL NT PL RO SB SK SL SP ST SZ TR UK A: AB AR GG FE IN KZ IQ TR WS

Ropalopus clavipes is not present in Latvia; most recent published record was based on misidentified specimens of $R$. macropus (D. Telnov, personal communication, 5.3.2021).

\section{PAGE 258}

PRINTED:

gracilis gracilis Krynicki, 1832: 162 (Obrium) E: AL AU BH BU CR CT CR CZ GE GR HU IT LA LT MC MD ME PL RO SB SK SL ST UK A: GG IS SY TR

MUST BE:

gracilis gracilis Krynicki, 1832: 162 (Obrium) E: AL AU BH BU CR CT CR CZ GE GR HU IT LA LF MC MD ME PL RO SB SK SL ST UK A: GG IS SY TR

Axinopalpis gracilis is not present in Lithuania and Latvia; most recent published records were based on misidentified specimens of Obrium brunneum [V. Tamutis and D. Telnov, personal communications, 5.3.2021].

\section{PAGE 261}

PRINTED:

campestris Faldermann, 1835: 435 (Callidium) Ei: CTi CZi GEi HUi MDi PLi ROi SKi STi UKi A: ABi ARi ANH ES FE GAN GUI HEB HEI HEN HUB HUN IN JA JIA JIL JIX KI KZ LIA MG NC NMO QIN SC SCH SHA SHN SHX TD TM UZ XIN XIZ YUN ZHE NARi ORR MUST BE:

campestris Faldermann, 1835: 435 (Callidium) Ei: CTi CZi FRi GEi HUi ITi LAi LTi MDi PLi ROi SKi STi SWi UKi A: ABi ARi ANH ES FE GAN GUI HEB HEI HEN HUB HUN IN JA JIA JIL JIX KI KZ LIA MG NC NMO QIN SC SCH SHA SHN SHX TD TM UZ XIN XIZ YUN ZHE NARi ORR

See: Pennacchio et al. [2016].

\section{PAGE 257}

PRINTED:

coelestis Semenov, 1911: 118 A: BEI FE GUA GUX HEB HEI HEN JIL NC SC SCH SHA SHN XIZ

MUST BE:

coelestis Semenov, 1911: 118 A: BEI FE GUA GUX HEB HEI HEN JA (Tsushima Is) JIL NC SC SCH SHA SHN $\mathrm{XIZ}$

24. PAGE 265

PRINTED: 
pallidipennis Heyden, 1887d: 321 (Gymnopterion) A: KI KZ UZ XIN

MUST BE:

pallidipennis Heyden, 1887b: 321 (Gymnopterion) A: KI KZ UZ XIN

\section{PAGE 282}

Purpuricenus sasanus Kadlec, 2006a was originally published as a species. Then its status was downgraded to subspecies rank by Sama [2010] and Rapuzzi, Sama [2014b] The species rank was restored in the Catalogue without comments. It was upgraded to species rank by Danilevsky et al. [2020].

26. PAGE 292

PRINTED:

griseus Fabricius, 1793: 261 (Cerambyx) E: AL AN AU BE BU BY CR CT CZ EN FI FR GE GR HU IT NT LA LS LT MC MD ME ND NR PL PT RO SB SK SL SP ST SV SZ TR UK A: ?AB ?AR CY ES FUJ GAN ?GG GUA GUI GUX HEB HEI HEN HUB JIL JIX KZ LIA MG NC NMO SHA SY TR WS XIN ZHE

MUST BE:

griseus Fabricius, 1793: 261 (Cerambyx) E: AL AN AU BE BU BY CR CT CZ EN FI FR GE GR HU IT NT LA LS LT MC MD ME ND NR PL PT RO SB SK SL SP ST SV SZ TR UK A: ?AB ?AR CY ES ?FUJ ?GAN ?GG ?GUA ?GUI ?GUX ?HEB ?HEI ?HEN ?HUB ?JIL ?JIX KZ ?LIA MG NC ?NMO ?SHA SY TR WS ?XIN ?ZHE \#74

All records of Acanthocinus griseus for China [Lin, Yang, 2019] were connected with other species, mostly with A. sachalinensis.

\section{PAGE 292}

PRINTED:

sachalinensis Matsushita, 1933a: 391 A: ES FE JA MG NC $\mathrm{SC}$

MUST BE:

sachalinensis Matsushita, 1933a: 391 A: CH ES FE JA MG $\mathrm{NC} \mathrm{SC}$

All records of $A$. griseus for China [Lin, Yang, 2019] were connected with other species, mostly with $A$. sachalinensis.

28. PAGE 300

PRINTED:

pilicornis pilicornis Fabricius, 1787: 148 (Saperda) A: ES FE HUB JIA JIL JIX NC NMO SC SCH SHA SHN ZHE

MUST BE:

pilicornis pilicornis Fabricius, 1787: 148 (Saperda) A: ES FE HUB JIA JIL JIX MG NC NMO SC SCH SHA SHN ZHE

29. PAGE 302

PRINTED:

dahli dahli C.F.W. Richter, 1821: pl. 12 (Saperda) E: AL AU BE BH BU BY CR CT CZ FR GE GR HU KZ MC MD ME RO SB SK SL SL SP ST ?SZ UK A: GG KZ

MUST BE:

dahli dahli C.F.W. Richter, 1820: pl. 12 (Saperda) E: AL AU BE BH BU BY CR CT CZ FR GE GR HU KZ MC MD ME RO SB SK SL SL SP ST ?SZ UK A: GG KZ

Agapanthia dahli (Richter, 1820) is the generally accepted date of description [Bousquet, 2016] for Richter [1820].
But Agapanthia dahli (Richter, 1821) was published by Aurivillius [1923], Winkler [1929], Bense [1995], Sama [2002], Sama et al. [2008], Sama [2011], Rapuzzi, Sama [2012] and others.

\section{PAGE 304}

PRINTED:

pesarinii Sama \& Rapuzzi, 2010: 177 E: TR A: TR

MUST BE:

pesarinii Sama \& Rapuzzi, 2010: 177 E: TR A: SY TR

\section{PAGE 304}

PRINTED:

violacea Fabricius, 1775: 187 (Saperda) E: AL AU BE BU

CT CR CZ FR GE GR HU IT LU MC MD ME NT PL PT

SB SK SL SP ST TR UK A: AB AR ES GG KZ TR

MUST BE:

violacea Fabricius, 1775: 187 (Saperda) E: AL AU BE BU

CT CR CZ FR GE GR HU IT LU MC MD ME NT PL PT

SB SK SL SP ST TR UK A: AB AR ES GG KZ TR WS

\section{PAGE 304}

PRINTED:

soror Kraatz, 1882c: 336 A: AF KI KZ TD ZU

MUST BE:

soror Kraatz, 1882c: 336 A: AF KI KZ TD UZ

33. PAGE 311

testacea testacea Fabricius, 1781: 235 (Saperda) E: AL AN AU BE BH BU BY CR CT CZ FR EN GE GR HU IT KZ LA LT LU MD ME NL PL RO SB SK SL SP ST SV SZ TR UK A: KZ

MUST BE:

testacea testacea Fabricius, 1781: 235 (Saperda) E: AL AN AU BE BH BU BY CR CT CZ FR EN GE GR HU IT KZ LA LT LU MD ME NL PL RO SB SK SL SP ST SV SZ TR UK A: KZ WS \#468

34. PAGE 332

PRINTED:

rugicollis rugicollis Chevrolat, 1852: 418 A: ANH FE FUJ GAN GUA GUI GUX HAI HEB HEN HKG HUB HUN JIA JIX LIA NC SC SCH SHA SHN SHX TAI XIZ YUN ZHE

MUST BE:

rugicollis rugicollis Chevrolat, 1852: 418 A: ANH FE FUJ GAN GUA GUI GUX HAI HEB HEN HKG HUB HUN JIA JIX JA LIA NC SC SCH SHA SHN SHX TAI XIZ YUN ZHE

\section{PAGE 337}

PRINTED:

capreolum Heyden, 1887d: 317

MUST BE:

capreolum Heyden, 1887b: 317

36. PAGE 338

PRINTED:

optatum kadyrbekovi Danilevsky, 1999b: 20 A: KZ

MUST BE:

optatum kadyrbekovi Danilevsky, 1999b: 20 A: KI

37. PAGE 341

PRINTED:

berytense breuning, 1964c: 31 (Beiruth) 
MUST BE:

berytense Breuning, 1964c: 31 (Beiruth)

\section{PAGE 345}

PRINTED:

elegans Kraatz, 1873a: 73 E: KZ ST UK A: KZ

MUST BE:

elegans Kraatz, 1873a: 73 E: KZ ST UK A: KZ WS

\section{PAGE 345}

PRINTED:

subcostatum Heyden, 1887d: 323

MUST BE:

subcostatum Heyden, 1887b: 323

\section{PAGE 355}

PRINTED:

scabricolle pseudosevangenses Lazarev, 2020a: 18 A: AR MUST BE:

scabricolle pseudosevangense Lazarev, 2020a: 18 A: AR

\section{PAGE 358}

PRINTED:

scopolii Herbst, 1784: 91 (Lamia) E: BU CR CZ HU ?MD PL RO SB SK UK

MUST BE:

scopolii Herbst, 1784: 91 (Lamia) E: BU CR CZ GR HU ?MD PL RO SB SK UK

\section{PAGE 358}

PRINTED:

tauricum pittinoi Pesarini \& Sabbadini, 2010: 185 E: GR

MUST BE:

tauricum pittinoi Pesarini \& Sabbadini, 2010: 185 E: GR TR

\section{PAGE 379}

\section{PRINTED:}

punctipennis punctipennis Mulsant \& Guillebeau, 1856: 103 E: AL AU BH BU BY CR CT CZ FR GE GR HU IT LU MD PL RO SK SL SP ST SZ TR UK graecus Pic, 19011: 52

punctipennis signatus Mulsant \& Rey, 1863: 163 E: ST A: AB GG TR

\section{MUST BE:}

punctipennis punctipennis Mulsant \& Guillebeau, 1856: 103 E: AL AU BH BU BY CR CT CZ FR GE GR HU IT LU MD PL RO SK SL SP ST SZ ?TR UK graecus Pic, 19011: 52

punctipennis signatus Mulsant \& Rey, 1863: 163 E: ST TR A: AB GG TR

\section{PAGE 424}

PRINTED:

regina Heyden, 1887d: 319 (Mallosia) A: TD

MUST BE:

regina Heyden, 1887b: 319 (Mallosia) A: TD

\section{PAGE 425}

PRINTED:

alexandrovi Plavilstshikov, $1915 \mathrm{c}$ : 109 (Oberea) A: FE JIL MUST BE:

alexandrovi Plavilstshikov, 1915c: 109 (Oberea) A: FE JIL infrequens Cherepanov, 1996: 136

Oberea alexandrovi var. infrequens Plavilstshikov, 1915c was an unavailable name, described from same population as the nominative form; "its author expressly gave it infrasubspecific rank" according to the Article 45.6.4. of ICZN. The name was published as available: Oberea alexandrovi ssp. infrequens Cherepanov, 1996.

\section{PAGE 427}

PRINTED:

ruficeps muchei Breuning, 1981a: 26 A: TD (Gissar Ridge, Anzon Pass)

MUST BE:

ruficeps muchei Breuning, 1981a: 26 A: TD (Gissar Ridge, Anzob Pass)

47. PAGE 436

PRINTED:

melichari Pic, 1952d: 693 ["Im hohen Nordkaukasus"]

MUST BE:

melichari Pic, 1914b: 10 ["Im hohen Nordkaukasus"]

\section{PAGE 442}

PRINTED:

geniculata geniculata Mulsant, 1862: 420 A: CY IN IQ IS JO

$$
\text { LE TR }
$$

MUST BE:

geniculata geniculata Mulsant, 1862: 420 A: CY IN IQ IS JO

$$
\text { LE SY TR }
$$

\section{PAGE 443}

PRINTED:

pustulata murina Marseul, 1870: 384 ["Sarepta"] E: ST A: KZ KI UK UZ

MUST BE:

pustulata murina Marseul, 1870: 384 ["Sarepta"] E: ST UK A: KZ KI UZ

\section{PAGES 447-448}

Three species Pogonocherus ehdenensis Sama et Rapuzzi, 2000, P. ovatoides Rapuzzi et Sama, 2014a and P. pilosipes Pic, $1907 \mathrm{c}$ were mistakenly placed in subgenus Pogonocherus (s.str.), but all belong to P. (Pityphilus) because of the absence of apical elytral spines.

\section{PAGES $447-448$}

Original genus name Pogonochaerus Gemminger, 1873 was missing for several Pogonocherus species names.

MUST BE:

pilosipes Pic, 1907c: 21 (Pogonochaerus) A: CH "Chine orientale",

pillosipes Pic, 1907c: 21 (Pogonochaerus) [incorrect original spelling]

eugeniae eugeniae Ganglbauer, 1891: 131 (Pogonochaerus) E: AL AU BH CR CZ GR IT RO

eugeniae taygetanus Pic, 1903a: 8 (Pogonochaerus) E: GR (Peloponnisos) rufescens Pic, 1917a: 6 (Pogonochaerus) icosiensis Peyerimhoff, 1918b: 143 (Pogonochaerus) vaulogeri Pic, 1927e: 1 (Pogonochaerus)

plasoni Ganglbauer, 1884: 526 (Pogonochaerus) E: BH CR GR

\section{PAGE 449}

PRINTED:

dorsalis Pascoe, 1858: 251 (Sthenias) A: CHQ FUJ GUI GUX HP NP SCH SD YUN ZHE ORR 
MUST BE:

dorsalis Pascoe, 1858: 251 (Sthenias) A: CHQ FUJ GUI GUX HP KA NP SCH SD YUN ZHE ORR

Anaches dorsalis (Pascoe, 1858) was recorded for Kashmir (as Pterolophia) by Breuning [1961d].

\section{PAGE 478}

PRINTED:

gilvipes gilvipes Faldermann, 1837: 290 (Anaetia) E: ST UK

A: AB AR GG TR

MUST BE:

gilvipes gilvipes Faldermann, 1837: 290 (Anaetia) E: ST UK

A: AB AR GG TR

\section{PAGE 478}

PRINTED:

praeustus praeustus Linnaeus, 1758: 399 (Leptura) E: AL

AU BE BH BU BY CR CT CZ DE EN FI FR GB GE GR HU IR IT LA LS LT LU MC MD ME NL NR NT PL PT RO SB SK SL SL SP ST SV SZ TR UK A: AB AR ES GG KZ MG SY TR WS \#464

inapicalis Pic, $1891 \mathrm{~b}: 37$

pilosus Geoffroy, 1785: 78 (Leptura) $[\mathrm{HN}]$

praecestus Dufour, 1843: 101 (Saperda)

ustulatus Hagenbach, 1822: 11 (Saperda)

MUST BE:

praeustus praeustus Linnaeus, 1758: 399 (Leptura) E: AL

AU BE BH BU BY CR CT CZ DE EN FI FR GB GE GR

HU IR IT LA LS LT LU MC MD ME NL NR NT PL PT RO SB SK SL SL SP ST SV SZ TR UK A: AB AR ES GG KZ MG SY TR WS

\#464

inapicalis Pic, 1891b: 37

pilosus Geoffroy, 1785: 78 (Leptura) $[\mathrm{HN}]$

praecestus Dufour, 1843: 101 (Saperda)

ustulatus Hagenbach, 1822: 11 (Saperda)

The name Saperda praecesta, Dufour, 1843: 101 (published as "S. praecesta. F.") was not a new name, but wrong spelling of Saperda praeusta Fabricius, and so unavailable.

\section{PAGE 556}

PRINTED:

Heyden, L.F.J.D. von 1887c: Vierter Beitrag zur Kenntniss der Coleopteren-Fauna der Amurländer. Deutsche Entomologische Zeitschrift 31: 297-304.

Heyden, L.F.J.D. von 1887d: xi. Beitrag zur Coleopteren-Fauna von Turkestan. Deutsche Entomologische Zeitschrift 31: $305-336$

MUST BE:

Heyden, L.F.J.D. von 1887a: Vierter Beitrag zur Kenntniss der Coleopteren-Fauna der Amurländer. Deutsche Entomologische Zeitschrift 31: 297-304.

Heyden, L.F.J.D. von 1887b: xi. Beitrag zur Coleopteren-Fauna von Turkestan. Deutsche Entomologische Zeitschrift 31: $305-336$

\section{PAGE 660}

PRINTED:

fomer

MUST BE:

former.

\section{References}

Aurivillius C. 1923. Cerambycidae: Lamiinae II. Pars 74 // Schen- kling S. (ed.). Coleopterorum Catalogus. Volumen XXIII. Cerambycidae II. Berlin: W. Junk. S.323-704.

Bense U. 1995. Longhorn beetles. Illustrated key to the Cerambycidae and Vesperidae of Europe. Weikersheim: Markgraf Verlag. $512 \mathrm{p}$.

Bousquet Y. 2016. Litteratura Coleopterologica (1758-1900): a guide to selected books related to the taxonomy of Coleoptera with publication dates and notes // ZooKeys. Vol.583. P.1-776.

Breuning S. 1961d. Cataloque des lamiaires du Monde (Col., Céramb.) 4. Lieferung. Tutzing: Museum G. Frey. P.183-284.

Breuning S. 1962a. Revision der Dorcadionini (Coleoptera, Cerambycidae) // Entomologische Abhandlungen und Berichte aus dem Staatlichen Museum für Tierkunde in Dresden. Bd.27. S.1-665.

Danilevsky M.L. 2002. Subspecific structure of Dorcadion (Dzhungarodorcadion) semenovi Ganglbauer, 1884 (Coleoptera, Cerambycidae) // Les Cahiers Magellanes. Vol.10 [2001]. P.1-18.

Danilevsky M.L. 2010c. Tribe Dorcadionini // I. Löbl, A. Smetana (eds.). Catalogue of Palaearctic Coleoptera. Vol.6. Stenstrup: Apollo Books. P.241-264.

Danilevsky M.L. 2014b. Additions and corrections to the new Catalogue of Palaearctic Cerambycidae (Coleoptera) edited by I. Löbl \& A. Smetana, 2010. Part. IX // Humanity space. International almanac. Vol.3. N.2. P.193-250.

Danilevsky M.L. 2017d. Several taxonomic notes on new descriptions of Turkish Dorcadion (Coleoptera, Cerambycidae) // Humanity Space. International Almanac. Vol.6. No.1. P.33-37.

Danilevsky M.L. 2020 (ed.). Catalogue of Palaearctic Coleoptera. Vol.6 (1), Chrysomeloidea I (Vesperidae, Disteniidae, Cerambycidae). Revised and updated edition. Leiden-Boston: Brill. P.i-xxii, 1-712.

Danilevsky M.L., Ghobari H., Faizi F., Nozari J. 2021. Three new taxa of longhorn beetles (Coleoptera: Cerambycidae) from Kurdistan province, west of Iran. // Zoology in the Middle East. Vol.67. No.1. P.42-47. [Published online: 08 Dec 2020].

Fuchs E., Breuning S. 1971. Die Cerambycidenausbeute der Anatolien expeditionen 1966-1967 des Naturhistorischen Museums, Wien // Annalen des Naturhistorischen Museums in Wien. Bd.75. S.435-439.

Lin M.-Y., Yang X.-K. (eds.). 2019. Catalogue of Chinese Coleoptera. Vol.9. Chrysomeloidea: Vesperidae, Disteniidae, Cerambycidae. Beijing: Science Press. i-xii, $575 \mathrm{p}$.

Löbl I., Smetana A. (eds.). 2010. Catalogue of Palaearctic Coleoptera. Vol.6. Chrysomeloidea. Stenstrup: Apollo Books. 924 p.

Özdikmen H. 2007. The longicorn beetles of Turkey (Coleoptera: Cerambycidae). Part I - Black Sea Region // Munis Entomology \& Zoology. Vol.2. P.179-422.

Özdikmen H., Turgut S. 2007. A review of the genus Musaria Thomson, 1864 (Coleoptera: Cerambycidae) in Turkey with zoogeographical remarks // Munis Entomology \& Zoology. Vol.2. No.2. P.511-516.

Pennacchio F., Marianelli L., Binazzi F., Francardi V., Paoli F., Griffo R., Roversi P. F. 2016. First interception of Trichoferus campestris (Faldermann, 1835) (Coleoptera Cerambycidae Cerambycinae) in Italy // Redia. Vol.99. P.59-62.

Plavilstshikov N.N. 1958a. Fauna SSSR. Zhestokrylye. T.XXIII. Vyp. 1. Zhuki-drovoseki. Ch.3. Podsemeistvo Lamiinae, ch.1. Moskva - Leningrad: Izdatel'stvo AN SSSR. $591+$ [1] p. [In Russian]

Rapuzzi P., Sama G. 2012. Contributo alla conoscenza dei Cerambycidae di Albania (Coleoptera, Cerambycidae) // Atti del Museo Civico di Storia Narurale Trieste. Vol.55. P.181-234.

Rapuzzi P., Sama G. 2014b. Revision of the Purpuricenus interscapillatus species-group and allied taxa (Coleoptera, Cerambycidae) // Fragmenta entomologica. Vol. 45 (2013). No.1-2. P.143-171.

Rejzek M., Sama G., Alziar G., Sadlo J. 2003. Host plants of longhorn beetles (Coleoptera: Cerambycidae) from the Balkan Peninsula, Asia Minor, and Iran (Part II) // Biocosme Mésogéen. Vol.19 (2002). No.3. P.161-162.

Richter C.F.W. 1820. Supplementa faunae insectorum Europae. Teil 1. Vratislaviae: Richter. $3+12$ p. +12 pls.

Sama G. 1982. Contributo allo studio dei coleotteri Cerambycidae di Grecia e Asia Minore // Fragmenta Entomologica. Vol.16. P.205-227. 
Sama G. 2002. Atlas of Cerambycidae of Europe and the Mediterranean area. Vol.1: northern, western, central and eastern Europe, British Isles and continental Europe from France (excl. Corsica) to Scandinavia and Urals. Zlín: Kabourek. 173 p.

Sama G. 2010. New Acts and Comments. Cerambycidae. P.49-58. // I. Löbl, A. Smetana (eds.). Catalogue of Palaearctic Coleoptera. Vol.6. Stenstrup: Apollo Books. 924 p.

Sama G. 2011. The Cerambycidae of Marganai and Montimannu (SW Sardinia) (Coleoptera) // Nardi G., Whitmore D., Bardiani M., Birtele D., Mason F., Spada L., Cerretti P. (eds.). Biodiversity of Marganai and Montimannu (Sardinia). Research in the framework of the ICP Forests network. Conservazione Habitat Invertebrati. Vol.5. P.543-552.

Sama G. 2011. [new taxon] // Sama G., Jansson N., Avc M., Sar kaya O., Co kun M., Kay T., Özdikmen H. Preliminary report on a survey of the saproxylic beetle fauna living on old hollow oaks (Quercus spp.) and oak wood in Turkey (Coleoptera: Cerambycidae)//Munis Entomology \& Zoology. Vol.6. No.2. P.826.

Sama G., Löbl I. 2010. [Western Palaearctic taxa, eastwards to Afghanistan, exluding Oman and Yemen and the countries of the former Soviet Union]. // I. Löbl, A. Smetana (eds.). Catalogue of Palaearctic Coleoptera. Vol.6. Stenstrup: Apollo Books. 924 p.

Sama G., Seddighi N., Talebi A.A. 2008. Preliminary note for a checklist of the Cerambycidae of Iran // Biocosme Mésogéen. Vol.25. P.101-126.

Toropov S.A., Milko D.A. 2013. Longhorns of the tribe Dorcadionini (Coleoptera, Cerambycidae) of Kazakhstan and Middle Asia. Bishkek: $104 \mathrm{p}$

Winkler A. 1929. Cerambycidae. Pars 9. Pars $10 / /$ Catalogus Coleopterorum regionis palaearcticae. Wien: A.Winkler Verlag. S.1135-1226. 